\title{
Influenza A/H1N1 in 2009: a pandemic in evolution
}

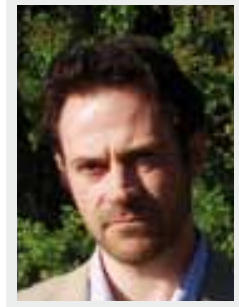

Tristan Clark, MRCP

Specialist Registrar in Infectious Diseases, Infectious Diseases Unit, University Hospitals of Leicester, Leicester, LE1 5WW, UK Tel.: +44 1162586952 Fax: +44 1162585067 tristan.w.clark@ uhl-tr.nhs.uk

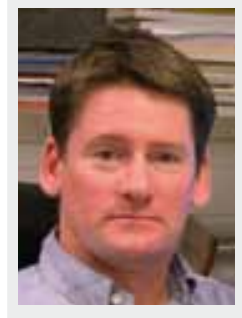

lain Stephenson, MA, FRCP Author for correspondence Senior Lecturer in Infectious Diseases, Infectious Diseases Unit, University Hospitals of Leicester, Leicester, LE1 5WW, UK Tel.: +441162586952 Fax: +44 1162585067 iain.stephenson@ uhl-tr.nhs.uk

\section{EXPERT
REVIEWS}

\author{
"Although seasonal vaccine production is well planned, rapid bulk \\ manufacture of pandemic vaccines of unknown growth characteristics \\ will be challenging."
}

Influenza has demonstrated its capacity to surprise with the emergence of a novel influenza A/H1N1 virus in North America. Air travel has a facilitated rapid intercontinental spread of the virus, threatening the next pandemic.

Current seasonal influenza $\mathrm{A} / \mathrm{H} 1 \mathrm{~N} 1$, $\mathrm{A} / \mathrm{H} 3 \mathrm{~N} 2$ and $\mathrm{B}$ viruses infect approximately $5-15 \%$ of the world's population each year, and are responsible for an estimated 500,000 excess deaths annually. Seasonal morbidity and mortality are highest during $\mathrm{A} / \mathrm{H} 3 \mathrm{~N} 2$ outbreaks, principally in young children, the elderly and those with chronic medical conditions. Effective annual immunization programs of seasonal vaccination generally focus on these three at-risk groups. Increased seasonal vaccine demand since the last pandemic has sustained an increase in vaccine manufacturing capacity that will be vital to a pandemic response.

Pandemics of influenza occur unpredictably when a novel virus with the capacity for person-to-person transmission emerges to which there is limited immunity in the population. During pandemics, agespecific attack rates may differ from those observed in seasonal outbreaks, with a greater impact on young adults. Reasons for the decline and replacement of existing influenza subtypes are uncertain. Experience from the 20th Century suggests that novel strains emerge from the animal reservoir of influenza A viruses following genetic reassortment between human and nonhuman strains. The pandemic H1N1 virus in 1918, H2N2 in 1957 and H3N2 in 1968 emerged to establish human lineages. Human-avian reassortant viruses caused the pandemics of 1957 and 1968, when avian viruses contributed the hemagglutinin gene to the pandemic strain. Genomic sequencing of the reconstructed 1918 pandemic H1N1 virus indicates its origin as an adapted avian virus without reassortment.

\section{"During pandemics, age-specific attack rates may differ from those observed in seasonal outbreaks, with a greater impact on young adults."}

Influenza as a disease of pigs was first described during 1918 when outbreaks of respiratory disease occurred simultaneously in humans and swine herds living and working in close proximity. Since then, occasional isolation of swine influenza viruses from humans with respiratory illness has confirmed that sporadic human infection can occur. In general, cases have been limited to those with occupational swine exposure. However, a pandemic alert was raised in 1976 when swine H1N1 caused an outbreak of respiratory illness with one fatality among 13 soldiers at a military base in Fort Dix (NJ, USA). No exposure to pigs was found and sero-epidemiological investigation identified up to 230 soldiers that had been infected, possibly via humanto-human transmission. Mass vaccination of the US public was initiated and subsequently halted amid reports of adverse reactions, media scepticism and the lack of pandemic activity [1].

Early analysis of the novel A/H1N1 outbreaks originating in Mexico and the USA during March-April 2009 is underway [101]. The A/H1N1 virus contains genetic segments from Eurasian and 
North American swine influenza strains, which in turn are triplereassortant viruses of human, avian and swine origin. All isolates are susceptible to neuraminidase inhibitors, but resistant to M2 inhibitors. Clinical symptoms are generally mild and similar to influenza-like illness, although gastrointestinal features appear more prominent than seasonal influenza. Clinical attack rates are highest in children and younger adults, with estimates of transmissibility $\left(\mathrm{R}_{0}: 1.4-1.6\right)$ significantly higher than observed in seasonal influenza and comparable to previous pandemics with case-fatality rates of approximately $0.4 \%$. The WHO has raised its pandemic alert level to Phase 5, indicating sustained person-to-person spread in several countries. It is uncertain what will transpire. A/H1N1 may disappear or acquire increased virulence as human passage continues. Observations from North America suggest that sustained community transmission is likely to increase. The upcoming summer may limit the impact in the northern hemisphere before it or a drifted A/H1N1 strain re-emerges during the winter to circulate alongside or replace existing influenza strains.

Efforts to strengthen pandemic preparedness plans over the last decade have been focused by the threat posed from highly pathogenic avian A/H5N1 viruses. Since the 1968 pandemic, anti-influenza drugs and better, more standardized vaccines are available. Neuraminidase inhibitors are highly effective in the treatment and prevention of seasonal influenza. Many countries have prepared antiviral drug stockpiles in preparation for pandemic influenza. In countries outside of North America, where there are limited numbers of imported $\mathrm{A} / \mathrm{H} 1 \mathrm{~N} 1$ infections, chemoprophylaxis of close contacts is reducing spread and may delay the onset of widespread activity until effective vaccines can be produced.

\section{6...chemoprophylaxis of close contacts is reducing spread and may delay the onset of widespread activity until effective vaccines can be produced."}

As the population is primed to influenza during interpandemic periods, immune responses to seasonal vaccines are fairly predictable, but the immune status of the population differs in a pandemic situation. At the onset of previous pandemics, younger adults were immunologically naive to the new strain, whereas older populations may have been primed by infections of related strains that circulated in earlier times. Global immune susceptibility to novel $\mathrm{A} / \mathrm{H} 1 \mathrm{~N} 1$ is expected, although the degree of cross-protection from infection with human A/H1N1 viruses circulating between 1918 and 1957, or since 1977 is unknown.

Although seasonal vaccine production is well planned, rapid bulk manufacture of pandemic vaccines of unknown growth characteristics will be challenging. Seasonal vaccines are trivalent, containing $15 \mu \mathrm{g}$ each of two influenza A (H1N1 and H3N2) and one $B$ strain. Traditional inactivated influenza vaccines are produced from virus grown in hens' eggs, and are of three formulations: whole virus, 'split product' or subunit 'surface antigen'. Most commercially available vaccines are split vaccines, produced from detergent-treated, highly purified virus or surface-antigen vaccines containing purified hemagglutinin and neuraminidase.
In the event of a pandemic declaration, vaccine demand will soar. Savings made by using monovalent rather than trivalent vaccines would likely be offset by the need for a two-dose schedule, higher antigen content and potential difficulties with production of egg-grown viruses. The use of mammalian cell lines such as Madin-Darby canine kidney cells or Vero cells to culture influenza virus for vaccines may increase flexibility at times of heightened demand. Immunopotentiating adjuvants or whole-virus vaccine formulation may increase immunogenicity, allowing reduction in dose content, enabling efficient use of limited antigen supplies. Alternatives to egg-derived or cell-cultured hemagglutinin is the production of antigen in modern expression systems that facilitate rapid antigen production, including: recombinant engineered baculovirus in insect cells, protein expression in plant cells and recombinant plasmids introduced into bacteria. However, the use of these technologies for clinical vaccines is unlicensed, and secondary manufacturing constraints (e.g., vial filling, needle and syringe supply, and requirement for adjuvantation) have not been addressed. In addition, potential difficulties with recombinant vaccines include the use of uncleaved hemagglutinin and differences in glycosylation that may affect immunogenicity.

"...neutralization assays may be more appropriate for the assessment of pandemic vaccines as hemagglutinin inhibition may be insensitive to nonhuman influenza strains."

New influenza vaccines must induce protective immunity and satisfy certain criteria, based on seroconversion, seroprotection and rises in antibody titers, set by the European Union Committee for Human Medicinal Products and the US FDA. As protective correlates of immunity for seasonal influenza are recognized for hemagglutinin inhibition and single-radial hemolysis antibody, these measures are used to licence seasonal vaccines. However, neutralization assays may be more appropriate for the assessment of pandemic vaccines as hemagglutinin inhibition may be insensitive to nonhuman influenza strains. However, as no correlates of protection for neutralizing antibody are recognized, this poses difficulties for pandemic vaccine licensure.

Regulatory approval for pandemic-specific vaccines may need to be granted during an evolving pandemic. The European approach has been to develop 'core dossiers' that contain preclinical and safety data pertinent to existing influenza vaccines. Dossiers are submitted by manufacturers to the EMEA in advance, and the strain or subtype variation added once the pandemic strain is identified. However, as the quantity of $\mathrm{A} / \mathrm{H} 1 \mathrm{~N} 1$ antigen and the number of doses or requirement for adjuvantation to induce acceptable immune responses is unknown, it is unclear how applicable this approach will be. As fast-track approval must not compromise safety, robust postmarketing surveillance is an integral part of licensing.

What information is available from previous pandemics or experience with avian influenza vaccine candidates? In 1976-1977, the emergence of A/New Jersey/76 (swine H1N1) afforded the opportunity to conduct vaccine trials in both immunologically naive 
and primed populations [2]. A series of whole-virus vaccine studies revealed differences between naive populations (those $\leq 24$ years of age and unexposed to previous H1N1 strains) and primed populations ( $>24$ years old). In naive subjects, if one dose of vaccine was administered, large doses of hemagglutinin $(>60 \mu \mathrm{g})$ were required. However, if two doses of vaccine were given, lower doses $(<5 \mu \mathrm{g})$ were required. Whole-virus vaccine was significantly more immunogenic than subunit or split-product vaccines. In primed subjects, as is the case during interpandemic periods, no differences in immunogenicity between whole-virus vaccine and subunit/splitproduct vaccines was observed. However, whole-virus vaccine was associated with increased reactogenicity, particularly in children, who developed febrile complications even with low doses.

\section{"The need for pandemic A/H1N1 vaccine must be balanced with seasonal vaccine production."}

Development of vaccines against $\mathrm{H} 5 \mathrm{~N} 1$ proved initially challenging, as wild-type $\mathrm{H} 5 \mathrm{~N} 1$ viruses are lethal to eggs used to grow vaccine viruses. In addition, the use of highly pathogenic strains requires biocontainment production facilities. To generate safe vaccine seed viruses, highly pathogenic strains are attenuated and then combined with influenza viruses that grow well in eggs. Traditional inactivated vaccines produced from avian $\mathrm{H} 5$ and $\mathrm{H} 9$ viruses are disappointing [3-6]. Two doses of subvirion $\mathrm{H} 5 \mathrm{~N} 1$ vaccine containing up to $90 \mu \mathrm{g}$ antigen demonstrated relatively poor immunogenicity, despite the antigen content being 12-times that of seasonal influenza vaccines. Studies in adults have found that alum salts do not enhance immunogenicity of subvirion $\mathrm{H} 5$ vaccines containing low antigen content, although among children there is some evidence that alum-adjuvanted subunit $\mathrm{H} 5$ vaccines can enhance responses.

Although whole-virus H1N1 vaccines produced in 1977 were superior to subunit vaccines in naive subjects, they were associated with febrile events. Cell culture and egg-derived whole-virus $\mathrm{H} 5 \mathrm{~N} 1$ vaccines are well tolerated in trials and demonstrate strong immunogenicity [7,8]; however, their use in children is limited. An important development from pandemic vaccine studies is the finding that oil-in-emulsion squalene-based adjuvants, such as MF59 or AS03, significantly enhance immune responses compared with nonadjuvanted vaccines. This permits dose sparing, which is essential for increasing the stock of available vaccine [5-7]. Such adjuvants are associated with increased local reactions, although these are generally mild and considered acceptable for a pandemic vaccine. Oil-in-emulsion adjuvants also broaden the humoral response by inducing neutralizing antibodies that are cross-reactive to vaccine virus drift variants. As priming with MF59-adjuvanted $\mathrm{H} 5$ vaccine induces cross-reactive memory $\mathrm{B}$ cells that can be mobilized many years later by boosting with antigenic $\mathrm{H} 5$ variant vaccine, it has been proposed that some of the population could receive a prepandemic vaccine to reduce delays associated with the production of pandemic-specific vaccine [9].

In order to produce a vaccine against $\mathrm{A} / \mathrm{H} 1 \mathrm{~N} 1$, a seed strain must be produced. Influenza viruses can be manipulated to produce strains that retain the novel surface antigens, but are suitable for rapid growth in eggs. Reverse-genetic systems for this purpose are established in a number of WHO-affiliated laboratories and are reliable for the manipulation of $\mathrm{H} 5 \mathrm{~N} 1$ viruses; however, experience with other subtypes is limited. As intellectual property rights surround this technology, manufacturers may need appropriate licences. It should take 3-6 weeks following receipt of wild-type virus to the release of an appropriate vaccine virus, and a further 6 months to produce significant quantities of pandemic-specific vaccine. Cell culture could increase flexibility over egg-grown virus and reduce delays if wild-type viruses can be used in vaccine production. Current events suggest an urgent need to undertake clinical assessment of $\mathrm{A} / \mathrm{H} 1 \mathrm{~N} 1$ vaccines in children, adults and the elderly to identify the amount of antigen and dose-schedule required to induce antibody responses.

The need for pandemic A/H1N1 vaccine must be balanced with seasonal vaccine production. The inclusion of novel A/H1N1 into the seasonal vaccine as an addition to, or replacement for, one of the existing components has been suggested. However, this approach poses challenges, as seasonal vaccines are licensed as trivalent products, given by a single administration of $15 \mu \mathrm{g}$ per strain. A pandemic-specific H1N1 vaccine may require two doses, adjuvantation or increased antigen content, creating difficulties in coadministration with seasonal strains. Furthermore, groups targeted for pandemic and seasonal vaccination may differ, as children and key workers are likely to be prioritized for pandemic vaccine.

\section{"...experience of mass vaccination with pandemic-specific vaccine is limited."}

Therefore, pandemic-specific A/H1N1 vaccine will be produced, at least initially, as a monovalent vaccine. This may affect the production capacity and availability of seasonal vaccines for 2009/2010. A WHO declaration of Phase 6 is likely to be accompanied by guidance on pandemic vaccine strain selection and production. Until official recommendations, manufacturers may have to make commercial decisions regarding their supplies. Some industrialized countries have advance purchase agreements for pandemic-specific vaccines, and activation of such contracts may reduce supplies to other countries. Even if full production capacity is directed towards monovalent pandemic vaccine, the total number of available doses that can be produced will not meet global demand.

Although annual seasonal vaccines are widely distributed, experience of mass vaccination with pandemic-specific vaccines is limited. Following the Fort Dix A/H1N1 outbreak, the US National Influenza Immunization Program was established to vaccinate the US adult population [1]. The US Federal government was forced to assume liability and indemnify the vaccine manufacturers before product delivery. Between October and December 1976, over 40 million people were immunized with monovalent A/New Jersey/76 (swine H1) whole-virus or bivalent A/New Jersey/76 and A/Victoria/75 (H3N2) split vaccine. The campaign was halted in December 1976, as an apparent association between vaccination and Guillain-Barré syndrome (GBS) 
was observed, with over 500 reported cases and 25 deaths [1]. Subsequent analysis suggested a vaccine-associated risk of GBS of 4.9-5.9 per million within 6 weeks following vaccination, contrasting with a low relative-risk of GBS of 0.6-1.4 per million following seasonal vaccination between 1978 and 1988 [10,11]. It remains uncertain whether this represents a true risk of GBS unique to the 1976 swine influenza vaccine, or the intensive surveillance that accompanied the program.

In conclusion, $\mathrm{A} / \mathrm{H} 1 \mathrm{~N} 1$ threatens a new pandemic. Although it appears to cause mild disease, there is potential for significant medical and economic disruption. The optimum content for $\mathrm{A} / \mathrm{H} 1 \mathrm{~N} 1$ vaccines is unknown, but is likely that two doses will be needed. Pandemic-specific vaccine production has started, but will take 6-8 months before significant quantities are available. Regulatory approval of pandemic vaccine may require fast-tracking, but mass vaccination must have robust postadministration surveillance. The unpredictable nature of influenza and the future unknowns of the $\mathrm{A} / \mathrm{H} 1 \mathrm{~N} 1$ outbreak would suggest that planning for mass immunization would be prudent.

\section{Financial \& competing interests disclosure}

Iain Stephenson has received funding from manufacturers of influenza vaccines, including Novartis Vaccines and GSK for scientific research, and received consultancy or speakers fees and support for travel to scientific meetings from Novartis Vaccines, GSK and Baxter Vaccines. The authors have no other relevant affiliations or financial involvement with any organization or entity with a financial interest in or financial conflict with the subject matter or materials discussed in the manuscript apart from those disclosed.

No writing assistance was utilized in the production of this manuscript.

\section{References}

1 Sencer D, Millar JD. Reflections on the 1976 swine flu vaccination program. Emerg. Infect. Dis. 12, 29-33 (2006).

2 Parkman PD, Hopps HE, Rastogi SC, Meyer HM Jr. Summary of influenza virus vaccine trials in adults. J. Infect. Dis. 136, S722-S730 (1977).

3 Treanor JJ, Campbell JD, Zangwill KM, Rowe T, Wolff M. Safety and immunogenicity of an inactivated subvirion influenza $\mathrm{A}(\mathrm{H} 5 \mathrm{~N} 1)$ vaccine. N. Eng. J. Med. 354, 1343-1351 (2006).

4 Nicholson KG, Colegate AE, Podda A et al. Safety and antigenicity of nonadjuvanted and MF59-adjuvanted influenza A/Duck/ Singapore/97 (H5N3) vaccine: a randomised trial of two potential vaccines against $\mathrm{H} 5 \mathrm{~N} 1$ influenza. Lancet 357 , 1937-1943 (2001).
5 Atmar R, Keitel WA, Patel SM et al. Safety and immunogenicity of non-adjuvanted and MF59-adjuvanted influenza A/H9N2 vaccine preparations. Clin. Infect. Dis. 43 , 1135-1142 (2006).

6 Leroux-Roels I, Borkowski A, Vanwolleghem T et al. Antigen sparing and cross reactive immunity with an adjuvanted $\mathrm{rH} 5 \mathrm{~N} 1$ prototype pandemic influenza vaccine: randomized controlled trial. Lancet 370, 580-589 (2007).

7 Lin J, Zhang J, Dong X et al. Safety and immunogenicity of an inactivated adjuvanted whole-virus influenza (H5N1) vaccine: Phase I controlled trial. Lancet 368, 991-997 (2006).

8 Ehrlich H, Müller M, Oh HM et al. A clinical trial of a whole-virus $\mathrm{H} 5 \mathrm{~N} 1$ vaccine derived from cell culture. N. Eng. J. Med. 358, 2573-2583 (2008).
9 Galli G, Hancock K, Hoschler K et al. Fast rise of broadly cross reactive antibodies after boosting long lived memory B cells primed by an MF59-adjuvanted vaccine. Proc. Natl Acad. Sci. USA 106, 7962-7967 (2009).

10 Langmuir AD, Bregman DJ, Kurland LT, Nathanson N, Victor M. An epidemiologic and clinical evaluation of Guillain-Barré syndrome reported in association with the administration of swine influenza vaccines. Am. J. Epidemiol. 119, 841-879 (1984).

11 Haber P, DeStefano F, Angulo FJ et al. Guillain-Barré syndrome following influenza vaccination. JAMA 292, 2478-2481 (2004).

\section{Website}

101 WHO. Influenza A (H1N1) - update 41 (2009) www.who.int/csr/don/2009_05_29/en/ index.html 